\section{Tumor Necrosis Factor-associated Palmoplantar Pustular Psoriasis Treated with Interleukin 6 Blocker}

\section{To the Editor:}

Anti-tumor necrosis factor (anti-TNF) compounds are regarded as some of the most potent agents available for treatment of psoriasis vulgaris, with $80 \%$ of patients achieving at least a $75 \%$ reduction of their baseline skin lesions after 10 weeks of therapy ${ }^{1}$.

Palmoplantar pustular psoriasis (PPP) is a chronic inflammatory skin condition characterized by recurrent eruptions of sterile pustules on erythematous skin, hyperkeratosis, and fissures on the palms and soles. Successful treatment options are usually limited. PPP has long been regarded as a localized variant of pustular psoriasis. Some authors have proffered that this pathological condition may not represent so much a subtype of psoriasis as a drug hypersensitivity reaction, as in acute generalized exanthematous pustulosis ${ }^{2,3}$.

Increased expression of TNF has been identified as an important pathophysiological mechanism in different types of chronic inflammation, including psoriasis and psoriatic arthritis. Despite the evident efficacy of anti-TNF therapies in this setting, many have described pustular psoriasis occurring as an adverse event of these agents in patients without prior psoriasis, such as those receiving anti-TNF therapy for rheumatoid arthritis $(\mathrm{RA})^{2,3,4,5}$

We describe a patient with RA who developed PPP during treatment with 3 different anti-TNF agents, with persistence of the condition despite cessation of therapy, without resolution of the phenomenon until anti-interleukin 6 (IL-6) therapy for RA was started. Helsinki Committee approval was obtained for this study and the patient gave consent for publication of this report.

A 52-year-old woman, diagnosed with RA 10 years earlier, was first treated with disease-modifying antirheumatic drugs for 5 years. In 2010, treatment with adalimumab was initiated. During the treatment there was no improvement of articular symptoms, but she developed PPP on her right foot (Figure 1). Her medical history did not include risk factors for PPP such as diabetes mellitus, smoking, or thyroid disease. Treatment was discontinued, with some skin improvement but worsening of the arthritis, and so etanercept was initiated, in consideration of the observation that PPP may resolve when switching to another anti-TNF agent. During this treatment, the PPP became exacerbated and she was admitted to the dermatology department. There was no response to treatment with cyclosporine for 3 months and topical medications. Unfortunately, anti-TNF patch tests are not available in our institute and therefore were not performed. A skin biopsy showed intraepidermal pustules along with heavy infiltration of neutrophils in the upper Malpighi layer, spongiosis, and perivascular mixed

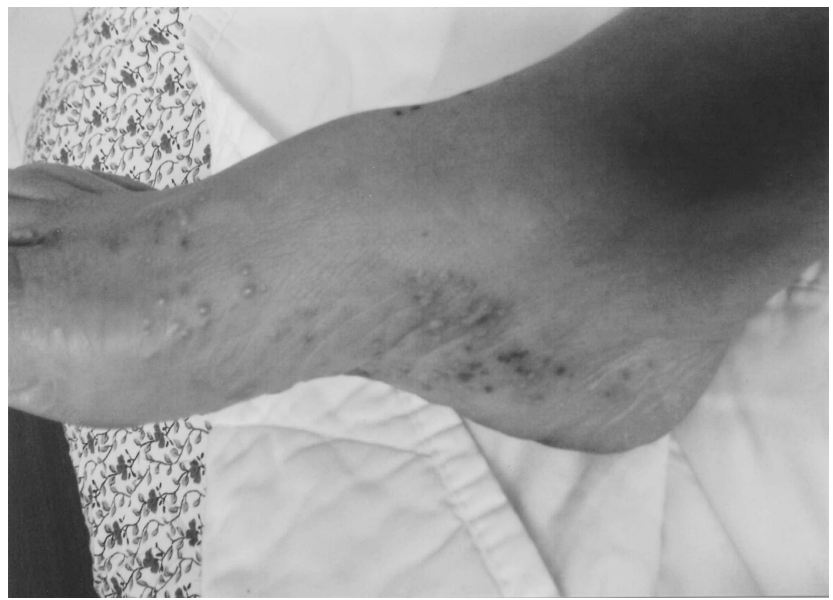

Figure 1. The patient's foot shows plantar pustulosis before treatment with tocilizumab. infiltrates of mononuclear cells and neutrophils, consistent with pustular psoriasis. Immunohistochemical studies were not performed on the biopsy. At that time, in coordination with the dermatologists, it was decided to attempt treatment with infliximab. Because of lack of response of both the arthritis and the skin lesions, treatment with infliximab was discontinued as well.

For the next 6 months the patient did not receive any new therapy and continued to suffer from severe PPP. Because she was in extreme anguish with very active arthritis along with the skin condition, it was decided to start tocilizumab $640 \mathrm{mg}$ monthly. Immediately with initiation of this therapy, and ahead of her joint response, it was noted that her PPP lesions improved, with a mild exacerbation just before the second tocilizumab treatment, and totally resolved by the third treatment (Figure 2), with no recurrence after 1 year of followup. Her arthritis has responded as well to the treatment, but not completely.

Generally, psoriasis is regarded as a $\mathrm{T}$ cell-mediated disorder with mainly $\mathrm{CD} 4+\mathrm{T}$ lymphocyte subsets present within the dermis. Interferon- $\alpha$-producing Th 1 cells are considered the principal pathogenic cells in this condition.

Few reports have addressed the contribution of IL- 6 to inflammation in psoriasis. IL-6 is produced in a regulated manner by keratinocytes, fibroblasts, and vascular endothelial cells as well as by leukocytes. Skin lesions of patients with psoriasis express a high level of IL-6 in comparison with nonlesional skin and with plaques remaining after treatment ${ }^{6}$. Therefore, IL-6 may directly contribute to the epidermal hyperplasia seen in psoriatic epithelium, as well as affecting the function of dermal inflammatory cells. Increased levels of IL-6 have also been reported in the plasma and serum of patients with active psoriasis ${ }^{7}$.

Fujishima and colleagues reported another indirect mechanism by which CD4+ T lymphocytes may cause inflammation by IL-6 in patients with psoriasis through Th17 cells ${ }^{8}$. These produce IL-17A, IL-17F, and IL-22 and may play an essential role in psoriasis. IL-17F acts as a selective neutrophil attractant in psoriasis ${ }^{8}$. IL-17F produced by $\mathrm{CD} 4+\mathrm{T}$ cells may cause inflammation in psoriasis partly through induction of IL-6 in keratinocytes $^{8}$. In agreement with this, recent studies of Th17 cells have already led to successful therapeutic strategies for psoriasis 9 .

Moreover, increased serum levels of IL-6 were found in patients with PPP. Tonsillectomy was associated with amelioration of PPP along with reduction of serum IL-6 level in these patients ${ }^{10}$. In vitro, exposing tonsillar mononuclear cells to streptococcal antigens resulted in increased production of IL-6 ${ }^{11}$

IL-6 is a cytokine involved in both the initiation and maintenance of the inflammatory and immunologic responses in certain autoimmune diseases. The level of IL-6 is elevated in many rheumatic diseases and IL-6 blockade has been shown to be beneficial in many of these, including RA

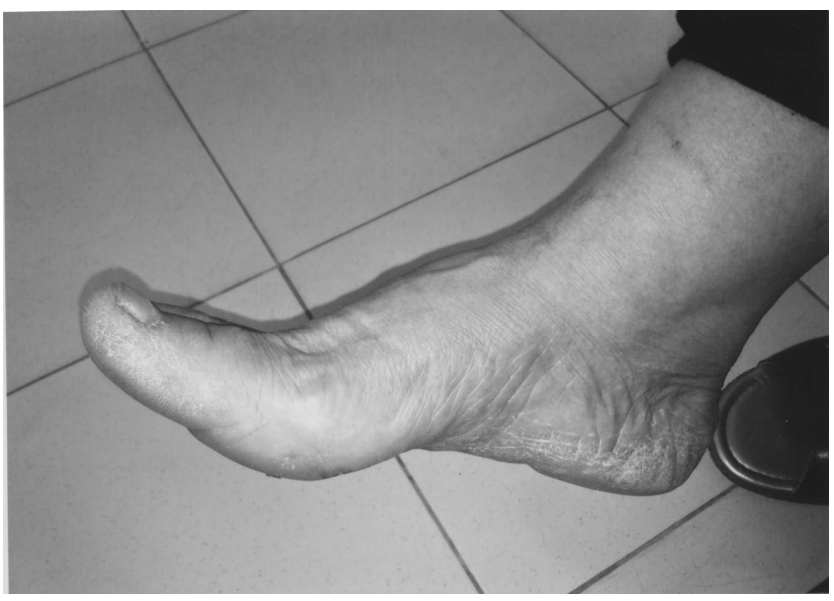

Figure 2. After treatment with tocilizumab. 
and juvenile idiopathic arthritis. There is no information on usefulness of anti-IL-6 therapy in psoriasis at present. We describe a case of PPP, a subgroup of psoriasis, that was an adverse event of anti-TNF treatment, which responded well to anti-IL-6 therapy. Further case reports and studies are warranted.

SAID YOUNIS, MD, Fellow, Rheumatology, Bnai Zion Medical Center, Haifa; DORON RIMAR, MD, Senior Physician, Rheumatology, Bnai Zion Medical Center, Adjunct Clinical Lecturer, Faculty of Medicine, Technion, Haifa; GLEB SLOBODIN, MD, Senior Physician, Rheumatology, Bnai Zion Medical Center, Senior Clinical Lecturer, Faculty of Medicine, Technion, Haifa; ITZHAK ROSNER, MD, Director, Rheumatology, Bnai Zion Medical Center, Associate Professor of Medicine, Faculty of Medicine, Technion, Haifa, Israel. Address correspondence to Prof. I. Rosner, Rheumatology, Bnai Zion Medical Center, PO Box 4940, Haifa, 31048 Israel. E-mail: rosneri@tx.technion.ac.il

\section{REFERENCES}

1. Reich K, Nestle FO, Papp K, Ortonne JP, Evans R, Guzzo C, et al. Infliximab induction and maintenance therapy for moderate-to-severe psoriasis: A phase III, multicentre, double-blind trial. Lancet 2005;366:1367-74.

2. Sfikakis PP, Iliopoulos A, Elezoglou A, Kittas C, Stratigos A. Psoriasis induced by anti-tumor necrosis factor therapy: A paradoxical adverse reaction. Arthritis Rheum 2005;52:2513-8.

3. Roux CH, Brocq O, Leccia N, Giacchero D, Breuil V, Albert C, et al. New-onset psoriatic palmoplantaris pustulosis following infliximab therapy: A class effect? J Rheumatol 2007;34:434-7.

4. Shmidt E, Wetter DA, Ferguson SB, Pittelkow MR. Psoriasis and palmoplantar pustulosis associated with tumor necrosis factor- $\alpha$ inhibitors: The Mayo Clinic experience, 1998 to 2010. J Am Acad Dermatol 2011 July 11. [E-pub ahead of print]
5. Pontikaki I, Shahi E, Frasin LA, Gianotti R, Gelmetti C, Gerloni V, et al. Skin manifestations induced by TNF-alpha inhibitors in juvenile idiopathic arthritis. Clin Rev Allergy Immunol 2012;42:131-4.

6. Grossman RM, Krueger J, Yourish D, Granelli-Piperno A, Murphy DP, May LT, et al. Interleukin 6 is expressed in high levels in psoriatic skin and stimulates proliferation of cultured human keratinocytes. Proc Natl Acad Sci USA 1989;86:6367-71.

7. Galadari I, Sheriff MO. Estimation of interleukin-6 level in psoriasis patients. Eur Ann Allergy Clin Immunol 2005;37:63-5.

8. Fujishima S, Watanabe H, Kawaguchi M, Suzuki T, Matsukura $\mathrm{S}$, Homma T, et al. Involvement of IL-17F via the induction of IL-6 in psoriasis. Arch Dermatol Res 2010;302:499-505.

9. Papp KA, Leonardi C, Menter A, Ortonne JP, Krueger JG, Kricorian G, et al. An anti-interleukin-17-receptor antibody for psoriasis. N Engl J Med 2012;366:1181-9.

10. Nakamura T, Oishi M, Johno M, Ono T, Honda M. Serum levels of interleukin 6 in patients with pustulosis palmaris et plantaris. J Dermatol 1993;20:763-6.

11. Murakata H, Harabuchi Y, Kataura A. Increased interleukin-6, interferon-gamma and tumour necrosis factor-alpha production by tonsillar mononuclear cells stimulated with alpha-streptococci in patients with pustulosis palmaris et plantaris. Acta Otolaryngol 1999;119:384-91.

J Rheumatol 2012;39:10; doi:10.3899/jrheum.120304 